IHTC16-

\title{
VORTEX INDUCED TRANSPORT PHENOMENA IN FLOWS PAST MICROPINS
}

\author{
Shigang Zhang ${ }^{1}$, Neil Cagney ${ }^{2}$, Stavroula Balabani ${ }^{2}$, Manish K Tiwari ${ }^{{ }^{*}}$ \\ Nanoengineered Systems Laboratory, UCL Mechanical Engineering, University College London, London, \\ WC1E 7JE, UK \\ UCL Mechanical Engineering, University College London, London, WC1E 7JE, UK
}

\begin{abstract}
The potential of vortex shedding behind cylindrical micropins to enhance mixing at the microscale is investigated. The confinement present in microchannels may prevent or delay the formation of vortices and weaken the performance of such a passive micromixer. Thus, the onset of vortex shedding in confined flows in micromixers need be elucidated to optimize mixing performance. In this work, the flow past a single cylindrical pin in microchannels with different levels of confinement was studied experimentally using a custom high-speed micro particle-image velocimetry ( $\mu$ PIV) system. This system can resolve the instantaneous velocity fields and the corresponding vortex shedding frequencies. Results show that the onset of vortex shedding is delayed by enhancing the vertical confinement for a channel width of three pin diameters. However, when vortex shedding is present, the vortex shedding frequency and corresponding Strouhal numbers $(S t)$ are greater in more confined channels for the same Reynolds number $(R e)$. Finite-Time Lyapunov Exponent (FTLE) analysis was performed on the acquired velocity fields to estimate the mixing performance. Preliminary results with a channel three pin diameters wide and three pin diameters high indicated that vortex shedding induces an enhancement in both the mixing in the wake and the mass flux across the centreline of the wake. We also discuss our results in the context of prior works focussing on exploiting vortex shedding for enhancing thermal transport.
\end{abstract}

KEY WORDS: Nano/Micro scale measurements, Micromixing, Vortex shedding, High speed $\mu$ PIV, FTLE

\section{INTRODUCTION}

Passive micromixers rely on the microchannel geometry to mix fluids by molecular diffusion and convection. Chaotic advection is a popular mixing mechanism for the (bio)chemical and pharmaceutical industries as it significantly improves the mixing performance which is inherently low in laminar, microscale flows [1]. Inserting obstacles, such as cylindrical pins or other 3D structures in a microfluidic channel can enhance mixing via chaotic advection as obstacles periodically change the direction of fluid particles, reducing the diffusion time [2]. At relatively high Reynolds number $\left(R e_{p i n}=U D / v\right.$, where $U$ is the mean velocity in the channel, $D$ is the obstacle diameter and $v$ is the kinematic viscosity), such obstacles can also shed periodic vortices, which can further increase mixing through stretching and folding of the species streams [3]. Recently, Alam et al. [4] and Santana et al. [5] numerically investigated the mixing performance of microfluidic channels with obstacles and the shape of obstacles was chosen as a cylindrical pin due to simple geometry and manufacturing convenience. However, they did not discuss the interaction between the vortices, from the channel walls and the cylinder wake. Griffith et al. [6] and Singha and Sinhamahapatra [7] argued, based on numerical simulations, that the transition to vortex shedding regime will be delayed when the channel walls are close to the cylinder. Thus, experimental data are required to support and analyse the effect of confinement between channel wall and cylindrical pin on the vortex shedding and mixing processes. Herein, a high-speed micro particle-image velocimetry ( $\mu$ PIV) method was developed to capture the vortex shedding dynamics in various confined microfluidic channels. Finite-Time Lyapunov Exponent (FTLE) analysis was applied as a 
post-processing method to estimate the mixing performance from the PIV data. The broad motivation of our work is to exploit vortex shedding to enhance both heat and mass (species) transport. In fact, given the analogous geometry and $R e_{p i n}$, it is well known that heat and mass transfer are governed by similar set of equations, thereby enhancement of one also leads to the enhance of the other [8]. In our past work we have already demonstrated that the flow past arrays of micropins [9], with associated vortex shedding, can strongly improve heat transfer. However, investigation of the transport phenomena in flows past a single, confined pins is the main intended contribution of the current work and as such is directly related to both heat and mass transfer.

\section{EXPERIMENTAL SETUP}

\subsection{Microfluidic Chip Fabrication}

Poly(methyl methacrylate) (PMMA) microfluidic channels with a single cylindrical pin and different levels of lateral confinement $w^{*}$ (the ratio of channel width $W$ and cylindrical pin diameter $D$ ) were fabricated by CNC micro-milling (Minitech Machinery, Georgia) and bonded by using a thermally-solvent assisted bonding technique [10]. The diameter of the cylindrical pin was $500 \pm 10 \mu \mathrm{m}$, the channel height ranged from $500-$ $1,500 \mu \mathrm{m}$ and the width varied between $1,000-2,000 \mu \mathrm{m}$, resulting in non-dimensional vertical confinements $h^{*}$ (the ratio of channel height $H$ and cylindrical pin diameter $D$ ) ranging from 1 to 3 and lateral confinement $w^{*}$ ranging from 2 to 4 . The total channel length was $8 \mathrm{~cm}$ and the distance from the inlet to the pin was $5 \mathrm{~cm}$ (i.e. 100 times larger than the pin diameter).

\subsection{Measurement Method and Experimental Conditions}

The experimental setup is shown schematically in Fig. 1. A custom $\mu$ PIV system was assembled comprising a fiber illuminator (150 W, Thorlabs, UK), a microscope assembly (Edmund Optics, UK) and a high-speed camera (Phantom V411, USA). The microfluidic chips were mounted on an XYZ stage (Thorlabs, UK). Distilled water was used as the working fluid at room temperature $\left(20^{\circ} \mathrm{C}\right)$. The flow was seeded with $1 \mu \mathrm{m}$ neutrally buoyant polystyrene particles (ThermoFisher, UK) and was driven by a syringe pump (Harvard Bioscience, USA) with flow rates ranging from 0.2 to $105 \mathrm{ml} / \mathrm{min}$. The $\mu \mathrm{PIV}$ measurements were obtained using bright field images, with the polystyrene particles acting as tracers. A $5 \mathrm{x}$ microscope objective was used resulting in a spatial resolution of $4.3 \mu \mathrm{m} / \mathrm{pixel}$. The frame rate ranged between $7.9 \mathrm{kHz}$ (time interval $=126 \mu \mathrm{s}$ ) and $36 \mathrm{kHz}$ (time interval $=27 \mu \mathrm{s}$ ) with respective maximum image sizes of $1024 \times 512$ pixels and $320 \times 256$ pixels. In each experiment, 5,000 images were acquired and were processed using standard PIV algorithms. This involved a multi-pass crosscorrelation procedure with a 50\% overlap and starting interrogation window of $128 \times 64$ pixels and ending with $32 \times 32$ pixels. Compared to conventional $\mu$ PIV systems, the high speed $\mu$ PIV system employed in this study offers considerable advantages. Renfer et al. [11] used a conventional $\mu$ PIV setup to study vortex shedding dynamics in microcavities with cylindrical micropin fin arrays. Due to the camera framerate limitation $(4 \mathrm{~Hz})$, the frequency of vortex shedding could not be determined from the acquired PIV images. Instead, a high-frequency dynamic pressure sensor was utilised to capture the vortex shedding frequency [3]. By contrast, the setup in this study allows both the instantaneous velocity fields and the corresponding vortex shedding frequencies to be captured, which will be discussed below. 
IHTC16-

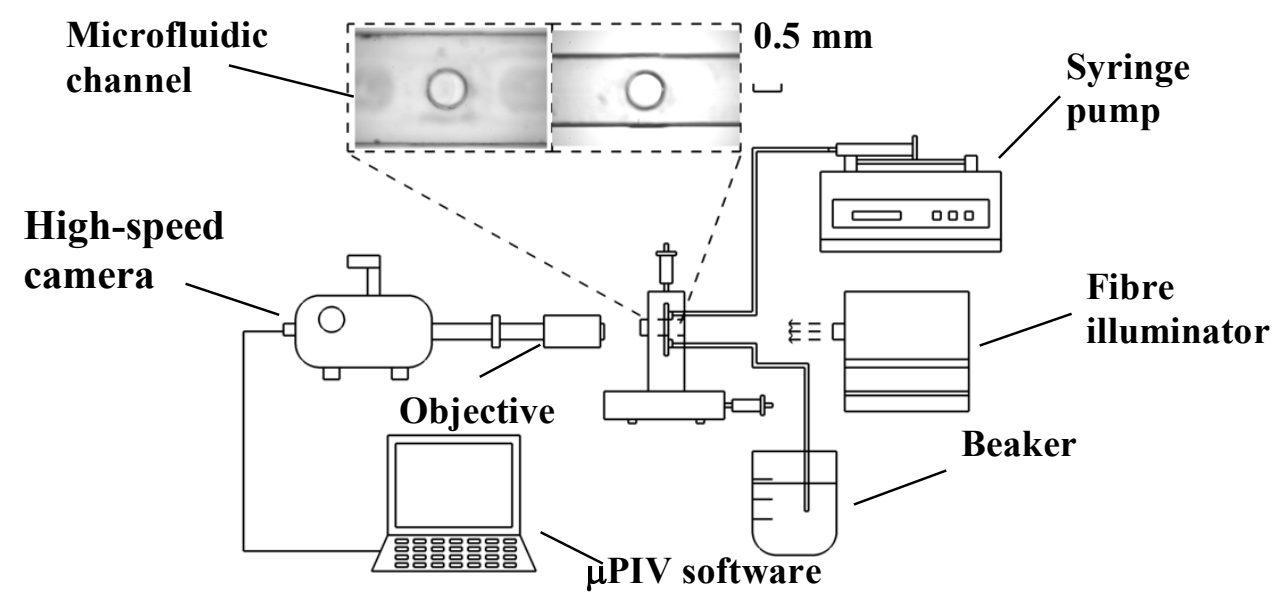

Fig. 1 Schematic of the experimental setup. Insets show a $500 \mu \mathrm{m}$ pin in two different width microchannels with: (a) $W=1500 \mu \mathrm{m}$ (b) $W=1000 \mu \mathrm{m}$.

\section{RESULTS AND DISCUSSION}

Selected instantaneous flow fields measured in microfluidic chips with non-dimensional vertical confinement $h^{*}$ $=2$ and lateral confinement $w^{*}=3$ are shown in Fig. 2. Vorticity fields are also shown in the same image. The vorticity contours show two elongated shear layers separating from the pin but no rolling up to form or shed vortices for $R e_{\text {pin }}$ values up to 160 due to wall confinement. Evidence of some flow periodicity was first observed when $R e_{\text {pin }}$ reached 180 and the shedding became more apparent and stronger when $R e_{\text {pin }}$ increased to 200 or 240. However, in a wider channel with $w^{*}>20$ and $h^{*}=7$ channel, vortex shedding behind a circular cylinder was detected at much lower $R e_{\text {pin }}$ of about 80 [12]. The results here demonstrate that strong confinement leads to a suppression of vortex shedding.
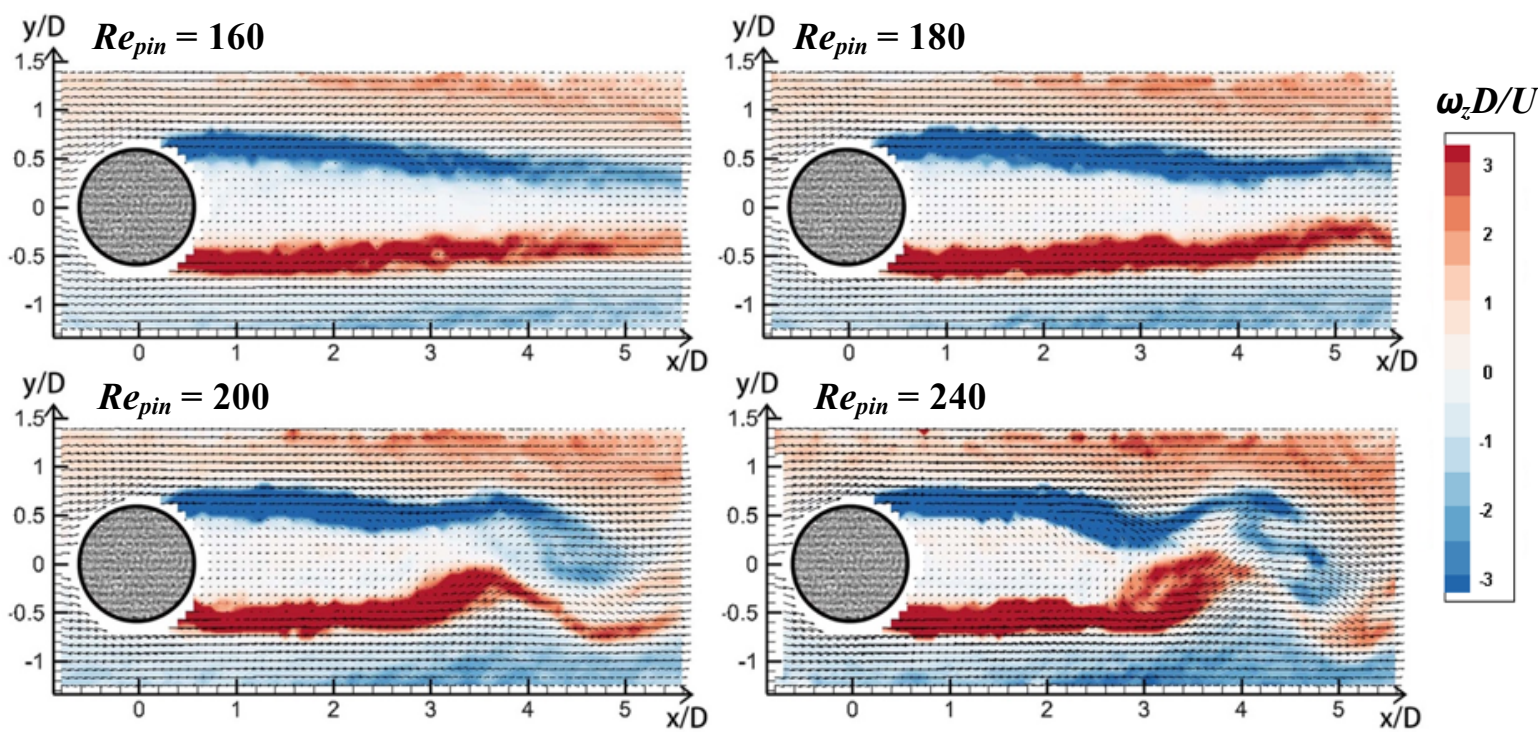

Fig. 2 Instantaneous normalized vorticity contours $\omega_{z} D / U$ superimposed onto the velocity vectors for a channel with $h^{*}=2$ and $w^{*}=3$, for different $R e_{\text {pin }}$.

Instantaneous contours of the transverse velocity, $V_{y}$, at two Reynolds numbers are shown in Fig. 3. Prior to the onset of vortex shedding (Fig. 3a), low values of the transverse velocity component are observed primarily close to the cylinder, and to less extent along the wake centerline $(y / D=0)$; however, once the 
vortex shedding sets on significant cross flow across the centerline occurs as demonstrated by the increased $V_{y}$ values in this region. The maximum absolute value of the transverse velocity along the wake centerline, $\left|V_{y \max }\right|$, was used to determine the onset of vortex shedding; as can be seen in Fig. 4a, $\left|V_{\text {ymax }}\right|$ is approximately zero at low $R e_{\text {pin }}$ before increasing dramatically beyond a critical value $\mathrm{i}$, which corresponds to the onset of vortex shedding. Fig. $4 \mathrm{a}$ indicates that the onset of vortex shedding is delayed by increasing vertical confinement (reducing $h^{*}$ ). For the case with $h^{*}=3$ and $w^{*}=3$, the vortices started shedding when $R e_{\text {pin }}$ reached 120 . The critical $R e_{\text {pin }}$ increases to 180 when $h *$ reduced to 2 . At even higher confinement $\left(h^{*}=1\right)$, no vortex shedding was observed until $R e_{p i n}=340$. This indicates that the channel walls suppress vortex shedding from the pin and delay the transition to the unsteady wake regime, in agreement with the numerical study of Griffith et al. [6]. The lateral confinement has a considerably weaker effect on the onset of vortex shedding, as can be seen in Fig. 4b, which compares $\left|V_{y \max }\right|$ for three values of $w^{*}$ for $h^{*}=2$. In all cases, the onset of vortex shedding occurs in the range $R e_{\text {pin }} \approx 160-200$. However, in this case the lateral confinement reduces the critical $R e_{p i n}$ for the onset of vortex shedding. This may be related to an increase in the maximum streamwise velocity past the cylinder with decreasing $w^{*}$.

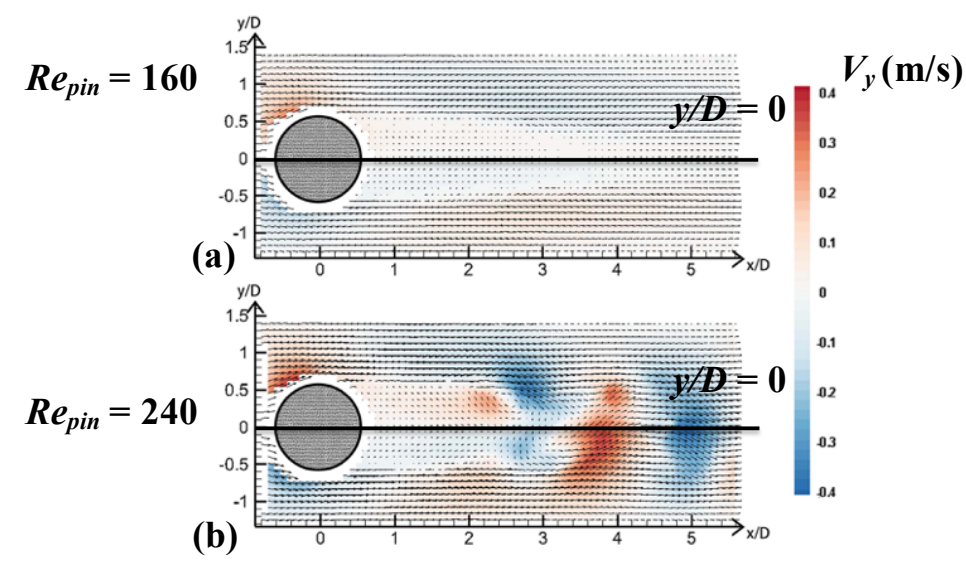

Fig. 3 Instantaneous transverse velocity $V_{y}$ contours superimposed onto the velocity vectors for a channel with $h^{*}=2$ and $w^{*}=3$ and for (a) $R e_{\text {pin }}=160$ (no vortex shedding) (b) $R e_{p i n}=240$ (with vortex shedding).

(a)

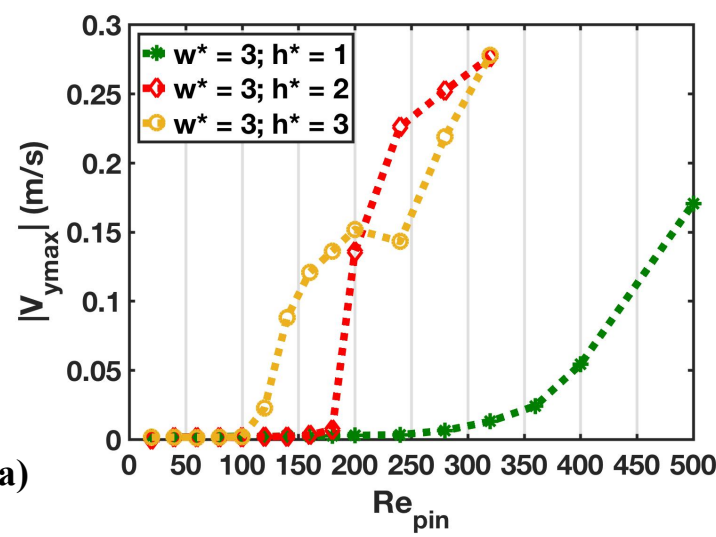

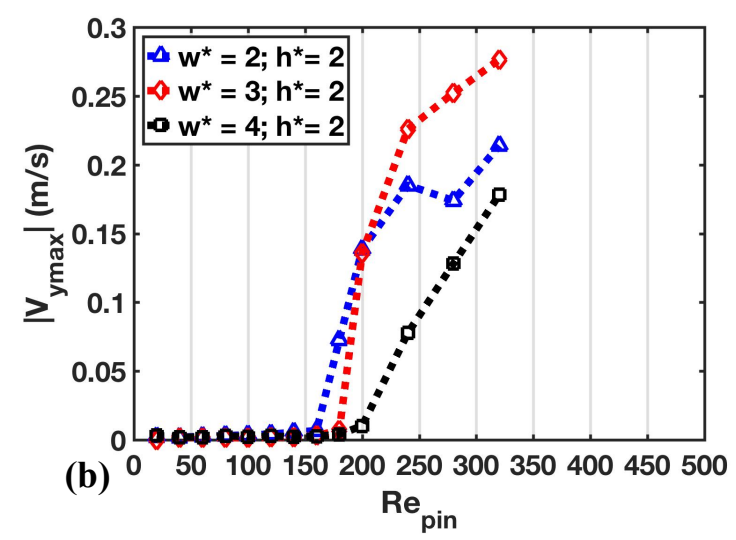

Fig. 4 The maximum absolute value of $V_{y}$ along $y / D=0$ position $\left(\left|V_{y \max }\right|\right)$ for different $R e_{\text {pin }}$ and for different (a) vertical confinement $h^{*}$ and (b) lateral confinement $w^{*}$.

\subsection{Recirculation Region Length}

Prior to the onset of vortex shedding, a vortex pair forms behind the pin and remains attached to the structure, forming a recirculation region [13]. Fig. 5 shows the axial velocity $V_{x}$ contours where red indicates positive values (i.e. flow directed away from the pin-downstream) and blue negative values (i.e. flow reversal). The white boundary (with velocity values close to zero), downstream of the pin, indicates the recirculation region. The 
recirculation region length, i.e. the distance between the end of recirculation region (where $V_{x}=0$ ) to the pin centre, in different confined channels and at different $R e_{\text {pin }}$ is plotted in Fig. 6. As $R e_{\text {pin }}$ is increased, the recirculation region length increases in all confined channels. Moreover, Fig. 6a clearly shows that for same $R e_{\text {pin }}$ and vertical confinement, the recirculation region length decreases with increased lateral confinement (reducing $w^{*}$ ). This is in agreement with the numerical study of Singha and Sinhamahapatra [7]. The vertical confinement was also found to affect the recirculation region length (Fig. 6b); however, the dependency of the recirculation region length on $h^{*}$ is not monotonic.
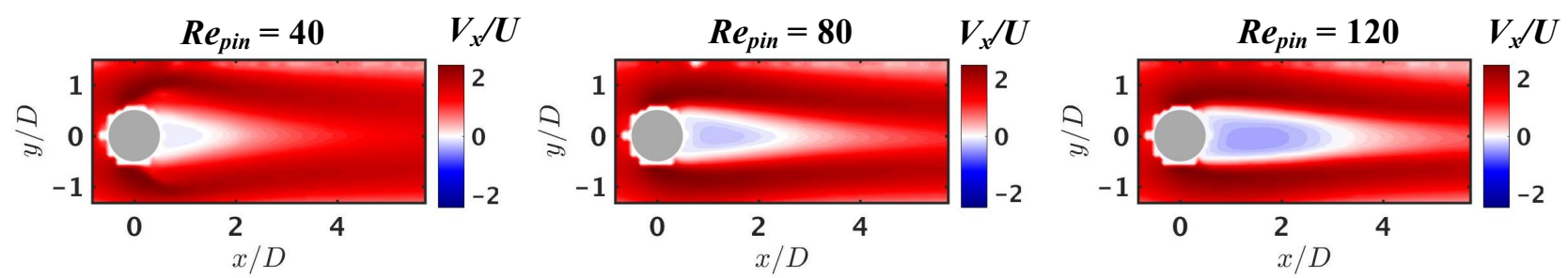

Fig. 5 Normalized axial velocity $V_{x}$ contours for a channel with $h^{*}=2$ and $w^{*}=3$ and for different $R e_{p i n}$. Velocities are normalized by the upstream velocity $\mathrm{U}$.

(a)

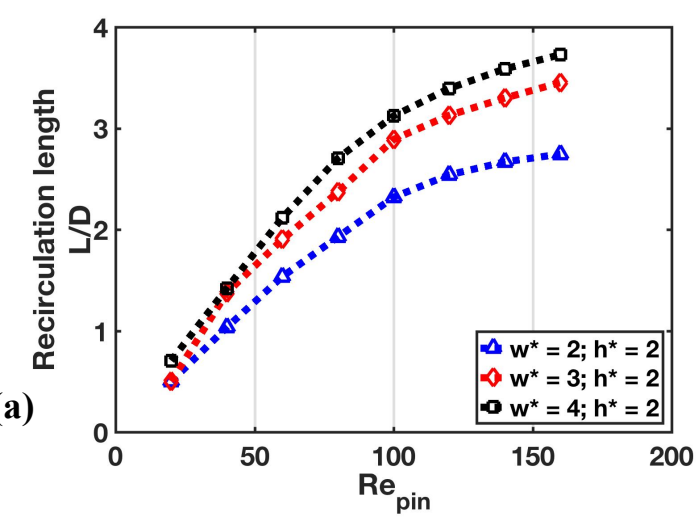

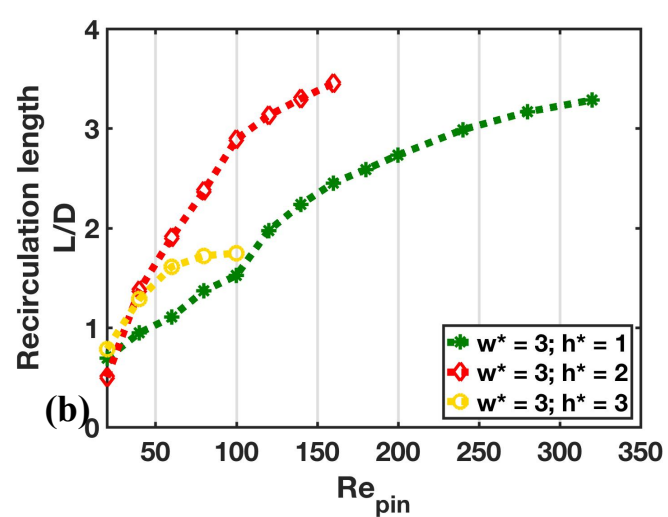

Fig. 6 Recirculation region length normalized by the pin diameter for different $R e_{p i n}$ and for different (a) lateral $w^{*}$ and (b) vertical confinements $h^{*}$.

\subsection{Vortex Shedding Frequency and Strouhal Number}

The shedding frequency is one of the most important properties of vortex shedding. As mentioned in section 2, the experimental system used in this study can simply acquire the vortex shedding frequencies without additional equipment. Fast Fourier transform (FFT) was applied to the instantaneous transverse velocity signal at a selected point downstream of the pin $(x / D=4, y / D=1)$. Fig. 7 a shows a typical transverse velocity signal extracted from 15,000 PIV fields obtained at a framerate of $36 \mathrm{kHz}$ for a channel with $w^{*}=3$ and $h^{*}=2$ at $R e_{\text {pin }}=180$. FFT analysis shows a clear peak (Fig. 7b) indicating a vortex shedding frequency of $373.5 \mathrm{~Hz}$. In order to obtain more reliable results, two additional points downstream of the wake were considered $(x / D=4, y / D=0 ; x / D=4, y / D=$ $-1)$ in each experiment and an average value of the vortex shedding frequency was taken. The FFT analysis for different $R e_{\text {pin }}$ at $w^{*}=3$ and $h^{*}=2$ channel are shown in Fig. 8, which illustrates that the shedding frequency increases with the increasing $R e_{\text {pin }}$ at the same channel. 

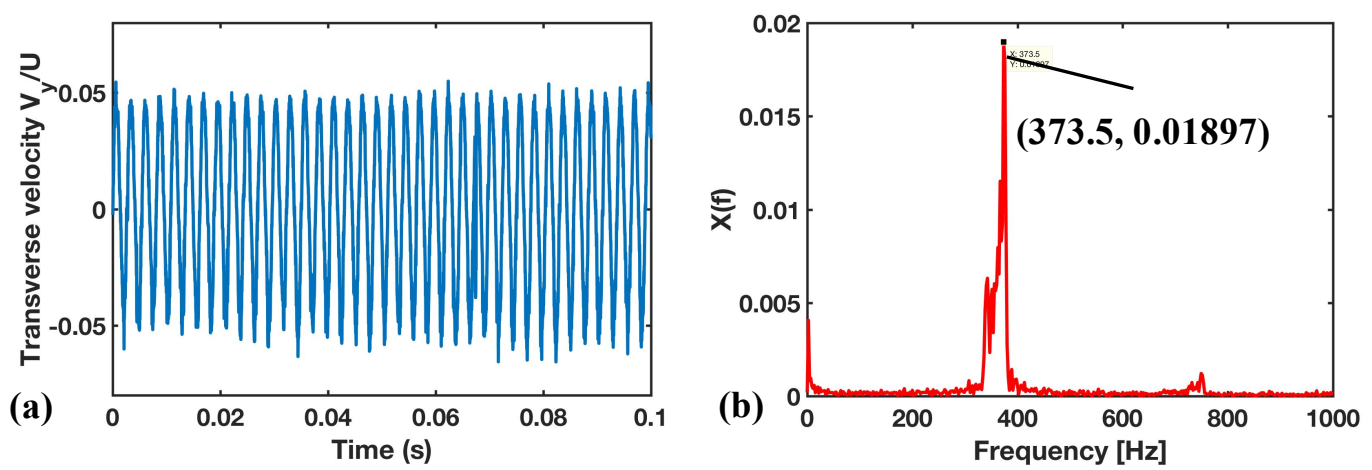

Fig. 7 (a) Instantaneous normalized transverse velocity $\left(V_{y} / U\right)$ signal at a selected point downstream of the pin in a channel with $w^{*}=3$ and $h^{*}=2$ at $R e_{p i n}=180$, (b) Amplitude spectrum of (a) showing a distinct vortex shedding peak.

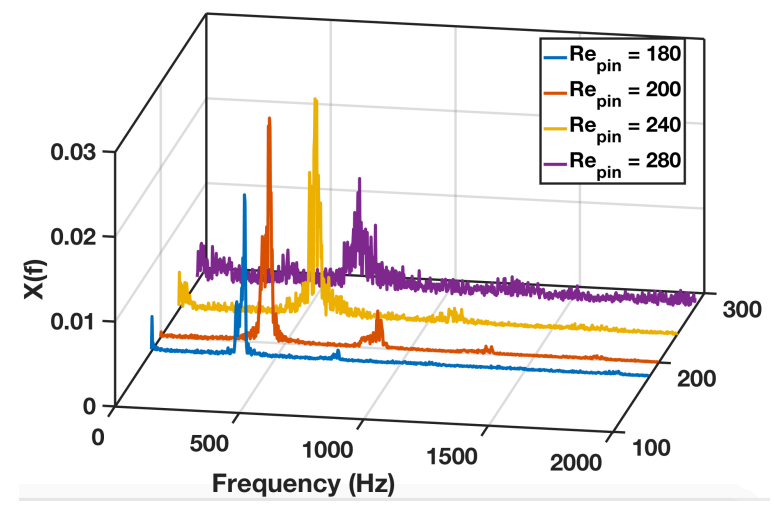

Fig. 8 Frequency spectra of the transverse velocity $\left(V_{y}\right)$ signals at different $R e_{p i n}$ for a channel with $w^{*}=3$ and $h^{*}=2$.

The Strouhal number $(S t)$ is a dimensionless number describing oscillating flow defined as $S t=f D / U_{\max }$, where $f$ is the frequency of vortex shedding, $D$ is the pin diameter and $U_{\max }$ is the maximum velocity upstream of the pin. Fig. 9 shows a set of normalized axial velocity profiles upstream of the cylinder $(x / D=-10)$ in a channel with $w^{*}$ $=2$ and $h^{*}=2$ at different $R e_{\text {pin }}$. Based on these upstream axial velocity profiles, the relationship between Strouhal numbers and $R e_{\text {pin }}$ for different confinements is shown in Fig. 10. It should be noted that for a channel with $w^{*}=$ 3 and $h^{*}=1$, vortices start shedding when $R e_{\text {pin }}$ reaches 340 . At this relatively high $R e_{\text {pin }}$, the frequency spectra did not produce a sufficiently clear peak to calculate $S t$. Thus, the results of the channel with $w^{*}=3$ and $h^{*}=1$ are not shown in Fig.10. The $S t$ tends to increase with increasing $R e_{p i n}$ and with reducing confinement. This is in agreement with the findings of Alfieri et al. [14] and Griffith et al. [6].

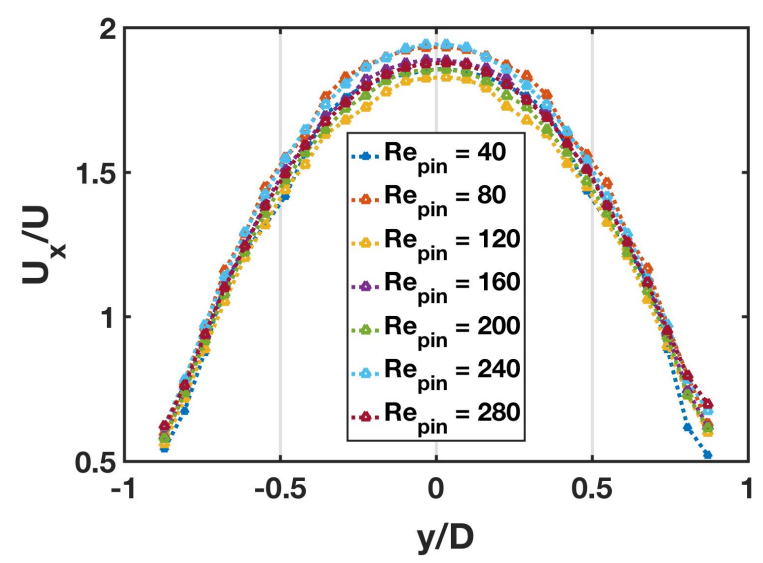

Fig. 9 Normalized axial velocity profiles upstream of the pin $(x / D=-10)$ obtained with a $w^{*}=2$ and $h^{*}=2$ channel at different $R e_{\text {pin }}$ 
(a)

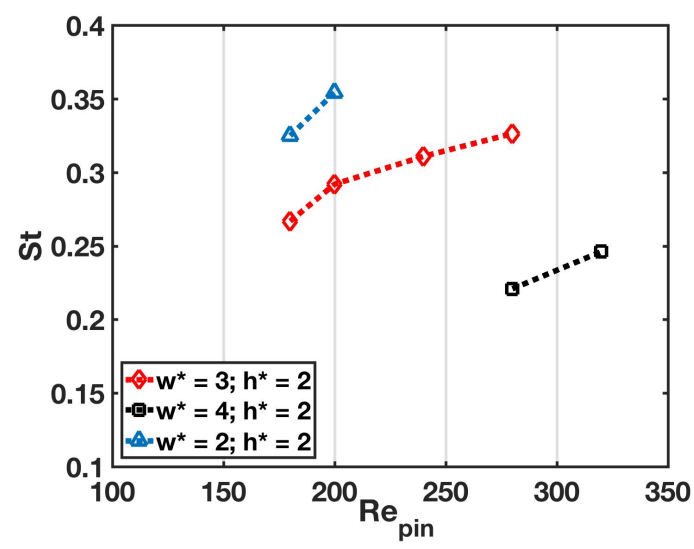

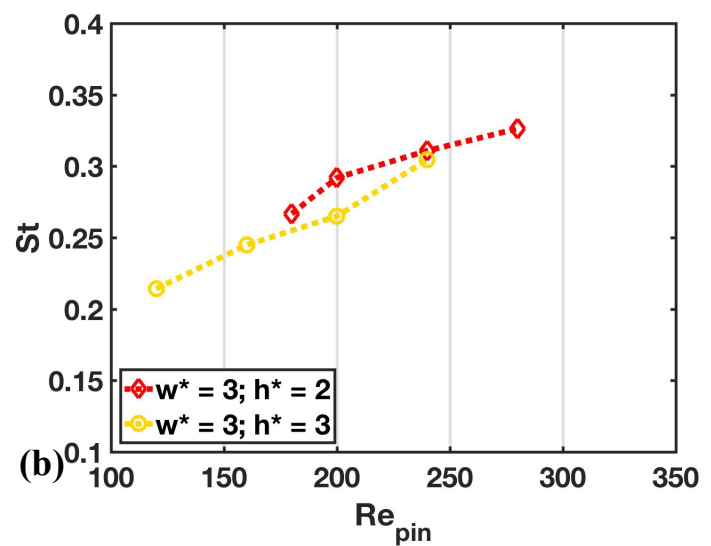

Fig. 10 The relationship between $S t$ and $R e_{\text {pin }}$ in channels with different (a) lateral confinement $w^{*}$ and (b) vertical confinement $h^{*}$

\subsection{Finite-Time Lyapunov Exponent (FTLE) Analysis}

In order to analyse the effect of vortex shedding on both the mixing and potentially heat transfer processes within the channel, we studied the Lagrangian characteristics of the flow. This is performed by numerically advecting tracers through the phase-average velocity fields in order to identify where individual regions of fluid will move to or have originated from. A commonly used Lagrangian measure of mixing is the Finite-Time Lyapunov Exponent (FTLE), which is a measure of the rate of fluid stretching[15]. FTLE fields can be computed by advecting the tracers either forward or backwards in time; elevated regions in the forward-time FTLE fields therefore indicate regions of the flow that will be stretched, while elevated regions in the backwards-time FTLE fields correspond to fluid regions that have already undergone intense stretching. The FTLE fields are an effective means of identifying coherent structures within a flow and characterising the regions where mixing is most efficient[16].
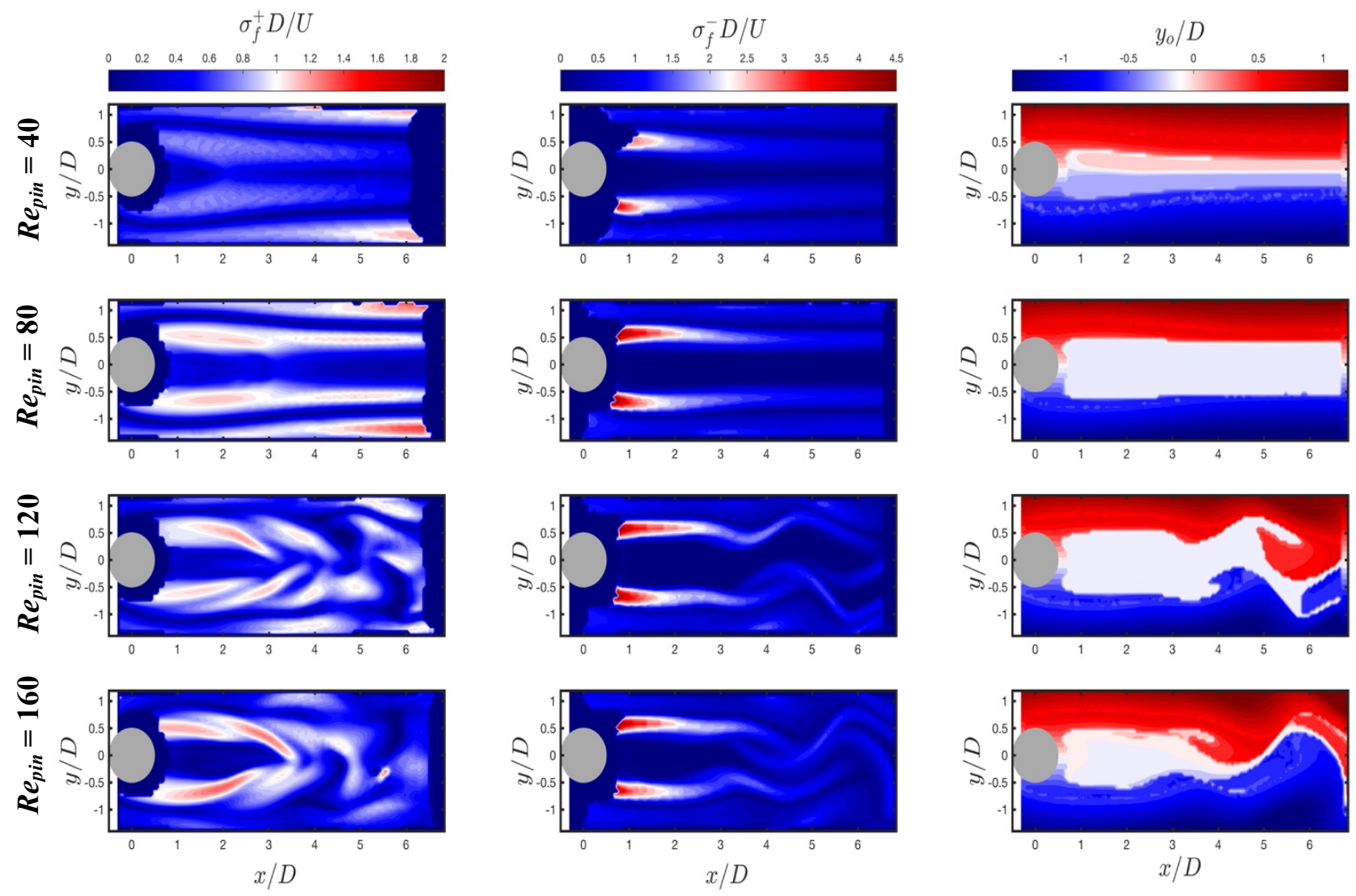
Fig. 11 FTLE results of the channel with $h^{*}=3$ and $w^{*}=3$ for different $\operatorname{Re}_{\text {pin }}$ with the forward-time FTLE (left column), the backward-time FTLE (middle column) and the tracers fields (right column), which indicate the transverse position from which each region of the fluid originated from. All data is shown for a single phase in the shedding cycle.

The normalised FTLE fields computed in forward- $\left(\sigma_{f}^{+}\right)$and backward-time $\left(\sigma_{f}^{-}\right)$are shown in Fig. 11 for a range of $R e_{p i n}$, for $w^{*}=3, h^{*}=3$. It can be seen that before the onset of vortex shedding $\left(R e_{p i n}=40\right.$ and 80$)$, the regions of stretching in forward and backward-time occur primarily at the channel walls and in narrow bands at $y / D \approx$ 0.75 , which approximately corresponds to the boundary between the recirculation region and the rest of the flow. However, once vortex shedding occurs, regions of intense stretching are distributed more evenly throughout the flow, as can be seen in the lower two rows of Fig. 11. This indicates that the vortex shedding induces an enhancement in mixing throughout the flow. The right column in Fig. 11 indicates the transverse origin, $y_{o}$, of tracers throughout the wake at a single phase. These 'tracer fields' are found by advecting the fluid tracers backwards in time over five shedding cycles (or the equivalent time in the cases without vortex shedding) and identifying the lateral position at which they entered the PIV domain. This is particularly relevant if the intention of the pin is to enhance heat transfer across the channel, and the aim is therefore to enhance mass flux from one side of the channel to the other. It can be seen that at low $R e_{p i n}$, before the onset of vortex shedding, there is negligible cross-wake mixing, i.e. fluid tracers on the upper side of the channel $(y / D>0)$ all originate on this side of the cylinder, $\left(y_{o} / D>0\right.$ also), and likewise for $(y / D<0)$. However, at higher $R e_{p i n}$, when vortex shedding is present, a significant proportion of the fluid near the centre of the wake originated close to the channel walls. This is indicative of enhanced cross-wake mixing. By measuring the percentage of material downstream $(a t x / D=6)$ that has advected across the wake centreline (i.e. the proportion of the fluid that has originated upstream on one side of the cylinder but by $x / D=6$ has now crossed to the opposite side of the wake) it is possible to quantify the magnitude of the cross-wake mixing. This ratio, which we term the cross-wake mixing coefficient, is shown in Fig. 12. It can clearly be seen that for higher $R e_{\text {pin }}$ after onset of vortex shedding, there is a significant increase in the cross-wake mixing coefficient, illustrating the ability of our approach to assess the improvements in mixing in microchannels due to the insertion of micropins. Based on the correlation between mass transfer and heat transfer [17][18], the improvement of mixing also indicates the heat transfer enhancement.

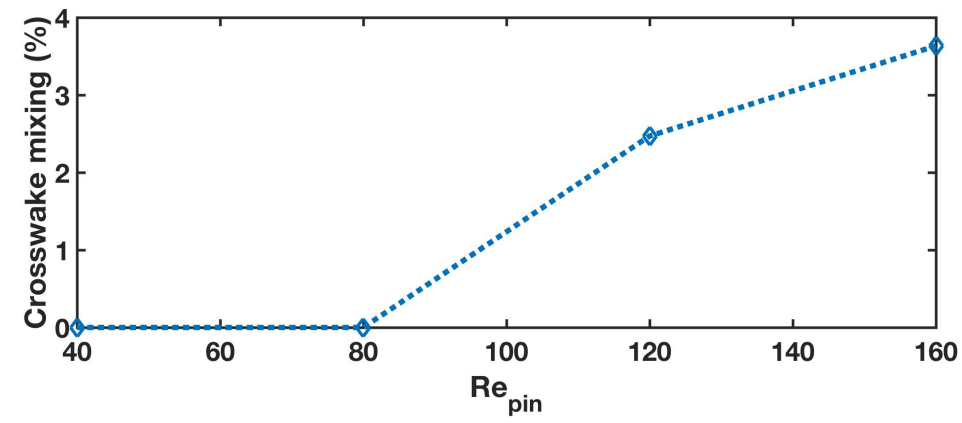

Fig. 12 The percentage of cross-wake mixing for a channel with $h^{*}=3$ and $w^{*}=3$ and for different $R e_{\text {pin }}$.

\section{CONCLUSIONS}

In this work, we propose to use micropins to significantly enhance mixing in microchannels through the effects of vortex shedding. A new experimental system was developed to fully resolve the transient flow in such fluidic chips by high speed PIV measurements in bright field mode. The study focused on a single cylindrical pin in order to explore the role of confinement effect on the onset of vortex shedding. The confinement suppressed/delayed shedding and affected the recirculation length and Strouhal number. Lagrangian analysis shows the results that vortex shedding enhances the mixing performance compared with channels without vortex shedding. Future work will focus on understanding the mixing performance in various confined channel. 


\section{ACKNOWLENGMENTS}

Partial funding by EPSRC projects EP/M029573/1 and EP/N024915/1 is gratefully acknowledged.

\section{REFERENCES}

[1] C.Y. Lee, W.T. Wang, C.C. Liu, L.M. Fu, Passive mixers in microfluidic systems: A review, Chem. Eng. J. 288 (2016) 146-160. doi:10.1016/j.cej.2015.10.122.

[2] N. Nguyen, Z. Wu, Micromixers-a review, J. Micromechanics Microengineering. 15 (2004) R1-R16. doi:10.1088/0960$1317 / 15 / 2 / \mathrm{R} 01$.

[3] A. Renfer, M.K. Tiwari, F. Meyer, T. Brunschwiler, B. Michel, D. Poulikakos, Vortex shedding from confined micropin arrays, Microfluid. Nanofluidics. 15 (2013) 231-242. doi:10.1007/s10404-013-1137-5.

[4] A. Alam, A. Afzal, K.Y. Kim, Mixing performance of a planar micromixer with circular obstructions in a curved microchannel, Chem. Eng. Res. Des. 92 (2014) 423-434. doi:10.1016/j.cherd.2013.09.008.

[5] H.S. Santana, J.L.S. Júnior, O.P. Taranto, Numerical simulations of biodiesel synthesis in microchannels with circular obstructions, Chem. Eng. Process. Process Intensif. 98 (2015) 137-146. doi:10.1016/j.cep.2015.10.011.

[6] M.D. Griffith, J. Leontini, M.C. Thompson, K. Hourigan, Vortex shedding and three-dimensional behaviour of flow past a cylinder confined in a channel, J. Fluids Struct. 27 (2011) 855-860. doi:10.1016/j.jfluidstructs.2011.02.007.

[7] S. Singha, K.P. Sinhamahapatra, Flow past a circular cylinder between parallel walls at low Reynolds numbers, Ocean Eng. 37 (2010) 757-769. doi:10.1016/j.oceaneng.2010.02.012.

[8] F.P. Incropera, T.L. Bergman, A.S. Lavine, D.P. DeWitt, Fundamentals of Heat and Mass Transfer, 2011. doi:10.1073/pnas.0703993104.

[9] F. Alfieri, M.K. Tiwari, A. Renfer, T. Brunschwiler, B. Michel, D. Poulikakos, Computational modeling of vortex shedding in water cooling of 3D integrated electronics, Int. J. Heat Fluid Flow. 44 (2013) 745-755. doi:10.1016/j.ijheatfluidflow.2013.10.004.

[10] A. Bamshad, A. Nikfarjam, H. Khaleghi, A new simple and fast thermally-solvent assisted method to bond PMMA-PMMA in micro-fluidics devices, J. Micromechanics Microengineering. 26 (2016) 65017. doi:10.1088/0960-1317/26/6/065017.

[11] A. Renfer, M.K. Tiwari, T. Brunschwiler, B. Michel, D. Poulikakos, Experimental investigation into vortex structure and pressure drop across microcavities in 3D integrated electronics, Exp. Fluids. 51 (2011) 731-741. doi:10.1007/s00348-0111091-5.

[12] P.J. Strykowski, K.R. Sreenivasan, On the formation and suppression of vortex "shedding" at low Reynolds numbers, J. Fluid Mech. 218 (1990) 71-107. doi:10.1017/S0022112090000933.

[13] S. Balachandar, R. Mittal, F.M. Najjar, Properties of the mean recirculation region in the wakes of two-dimensional bluff bodies, J. Fluid Mech. 351 (1997) S0022112097007179. doi:10.1017/S0022112097007179.

[14] F. Alfieri, M.K. Tiwari, A. Renfer, T. Brunschwiler, B. Michel, D. Poulikakos, Computational modeling of vortex shedding in water cooling of 3D integrated electronics, Int. J. Heat Fluid Flow. 44 (2013) 745-755. doi:10.1016/j.ijheatfluidflow.2013.10.004.

[15] N. Cagney, S. Balabani, Lagrangian structures and mixing in the wake of a streamwise oscillating cylinder, Phys. Fluids. 28 (2016). doi:10.1063/1.4945784.

[16] S.C. Shadden, F. Lekien, J.E. Marsden, Definition and properties of Lagrangian coherent structures from finite-time Lyapunov exponents in two-dimensional aperiodic flows, Phys. D Nonlinear Phenom. 212 (2005) 271-304. doi:10.1016/j.physd.2005.10.007.

[17] D. Kadylak, P. Cave, W. Mérida, Effectiveness correlations for heat and mass transfer in membrane humidifiers, Int. J. Heat Mass Transf. 52 (2009) 1504-1509. doi:10.1016/j.ijheatmasstransfer.2008.09.002.

[18] S.R. Gundlapally, V. Balakotaiah, Heat and mass transfer correlations and bifurcation analysis of catalytic monoliths with developing flows, Chem. Eng. Sci. 66 (2011) 1879-1892. doi:10.1016/j.ces.2011.01.045. 